\title{
Rifaximin modulates TRH and TRH-like peptide expression throughout the brain and peripheral tissues of male rats
}

Albert Eugene Pekary ${ }^{1,3,6^{*}}$ and Albert Sattin ${ }^{1,2,4,5}$

\begin{abstract}
Background: The TRH/TRH-R1 receptor signaling pathway within the neurons of the dorsal vagal complex is an important mediator of the brain-gut axis. Mental health and protection from a variety of neuropathologies, such as autism, Attention Deficit Hyperactivity Disorder, Alzheimer's and Parkinson's disease, major depression, migraine and epilepsy are influenced by the gut microbiome and is mediated by the vagus nerve. The antibiotic rifaximin (RF) does not cross the gut-blood barrier. It changes the composition of the gut microbiome resulting in therapeutic benefits for traveler's diarrhea, hepatic encephalopathy, and prostatitis. TRH and TRH-like peptides, with the structure pGlu-XPro- $\mathrm{NH}_{2}$, where " $\mathrm{X}$ " can be any amino acid residue, have reproduction-enhancing, caloric-restriction-like, anti-aging, pancreatic- $\beta$ cell-, cardiovascular-, and neuroprotective effects. TRH and TRH-like peptides occur not only throughout the CNS but also in peripheral tissues. To elucidate the involvement of TRH-like peptides in brain-gut-reproductive system interactions 16 male Sprague-Dawley rats, $203 \pm 6$ g, were divided into 4 groups ( $n=4 /$ group): the control (CON) group remained on ad libitum Purina rodent chow and water for 10 days until decapitation, acute (AC) group receiving $150 \mathrm{mg} \mathrm{RF/kg} \mathrm{powdered} \mathrm{rodent} \mathrm{chow} \mathrm{for} 24 \mathrm{~h}$ providing $150 \mathrm{mg} \mathrm{RF} / \mathrm{kg}$ body weight for $200 \mathrm{~g}$ rats, chronic (CHR) animals receiving RF for 10 days; withdrawal (WD) rats receiving RF for 8 days and then normal chow for 2 days.

Results: Significant changes in the levels of TRH and TRH-like peptides occurred throughout the brain and peripheral tissues in response to RF. The number of significant changes in TRH and TRH-like peptide levels in brain resulting from RF treatment, in descending order were: medulla (16), piriform cortex (8), nucleus accumbens (7), frontal cortex (5), striatum (3), amygdala (3), entorhinal cortex (3), anterior (2), and posterior cingulate (2), hippocampus (1), hypothalamus (0) and cerebellum (0). The corresponding ranking for peripheral tissues were: prostate (6), adrenals (4), pancreas (3), liver (2), testis (1), heart (0).
\end{abstract}

Conclusions: The sensitivity of TRH and TRH-like peptide expression to RF treatment, particularly in the medulla oblongata and prostate, is consistent with the participation of these peptides in the therapeutic effects of RF.

Keywords: TRH, Rifaximin, Medulla, Cortex, Prostate, Adrenal

\section{${ }^{*}$ Correspondence: albert.pekary@va.gov}

${ }^{1}$ Research Services, VA Greater Los Angeles Healthcare System, Bldg. 114,

Rm. 229B, 11301 Wilshire Blvd., Los Angeles, CA 90073, USA

Full list of author information is available at the end of the article

\section{Background}

Mental health and protection from a variety of agingrelated neurodegenerative disorders, such as autism, Attention Deficit Hyperactivity Disorder, Alzheimer's and Parkinson's disease, major depression, migraine and epilepsy, involve the gut microbiome and is mediated by the vagus nerve [1-4]. This is most evident in the behavioral abnormalities and GI symptoms of germ-free (GF) 
rodents $[4,5]$. The mechanisms underlying these effects include reduced levels of brain-derived neurotrophic factors in the cortex, hippocampus, and amygdala, and altered expression of genes encoding subunits of the glutamate and dopamine receptors [1]. Glutamatergic neurons are the most abundant excitatory class of nerves in the mammalian nervous system which requires co-release of the neuromodulatory thyrotropin releasing hormone (TRH) and TRH-like peptides to protect postsynaptic cells from the excitotoxic effects of excessive glutamate release [6-9]. The TRH/TRH-R1 receptor signaling pathway is an important mediator of brain-gut axis communication via the brain medulla oblongata and its associated TRH synthesizing neurons within the raphe pallidus, raphe obscura, and parapyramidal regions [10]. TRH and TRH-like peptides, with the structure pGlu-XPro- $\mathrm{NH}_{2}$ where " $\mathrm{X}$ " can be any amino acid residue, have reproductive, antidepressant, anxiolytic, analeptic, anorexic, and anti-aging effects [11].

TRH and TRH-like peptides occur not only throughout the CNS but also peripheral tissues, with very high levels in the rat and human prostate [11]. This is particularly noteworthy given the vulnerability of humans to prostatitis and prostate cancer [12]. Recent studies have implicated bacterial infections as potential causes of prostate diseases. The antibiotic rifaximin (RF), which does not cross the gut-blood barrier, is a standard treatment for traveler's diarrhea and hepatic encephalopathy. Its therapeutic potential in the treatment of other brain and urogenital disorders is currently being evaluated [12].

RF has anti-depressant and anxiolytic effects in both humans and rodents which are mediated, at least in part, by its ability to modify the composition of the gut microbiota [13-18]. Inadequate and/or irregulate sleep and poor nutrition contribute to obesity, alterations in the microbiome and the expression of gut hormones, including leptin and ghrelin, which have a profound effect on both appetite [19-23] and TRH and TRH-like peptide release $[24,25]$. Leptin and ghrelin also have mood altering effects [24, 25].

The present studies examine the effects of oral RF on TRH and TRH-like peptide levels in those brain regions, for example the medulla oblongata, and peripheral tissues which may play a role in the therapeutic effects of this gut-limited antibiotic [12].

\section{Methods}

\section{Animals}

"Young adult male Sprague-Dawley rats $(\mathrm{n}=16, \mathrm{SPF}$, Envigo, Indianapolis, IN) were used for all experiments. These animals were group housed ( 2 animals per cage) on wood shavings with a red plastic tube for play and shelter. Standard Purina rodent chow \#5001 and water were provided ad libitum during a standard one-week initial quarantine with $22 \pm 2{ }^{\circ} \mathrm{C}$ and $50 \pm 10 \%$ relative humidity; lights on: 6 am- $6 \mathrm{pm}$. Cages, water and bedding were changed every 3 days. All animals were weighed on the day of receipt and on the morning of each experiment. Initial body weights did not differ between experimental groups. Animals were randomized prior to the start of rifaximin treatment. Research was approved by the VA Greater Los Angeles Healthcare System Animal Care and Use Committee (IACUC Protocol \#030090-10) and conducted in compliance with the Animal Welfare Act and the federal statutes and regulations related to animals and experiments involving animals and adheres to principles stated in the Guide for the Care and use of Laboratory Animals, Eighth Edition, NRC Publication, 2011. All efforts have been made to minimize the number of animals used and their suffering. Animal was handled for 10 min per day for one month and then transferred from the Veterinary Medical Unit to the laboratory $12 \mathrm{~h}$ before the start of experiments to minimize the stress of a novel environment" [11]. "The American Veterinary Medical Association has concluded that decapitation without prior sedation 'is conditionally acceptable if performed correctly, and it should be used in research settings when its use is required by the experimental design and approved by the Institutional Animal Care and Use Committee" [26]. This study is reported in accordance with ARRIVE guidelines (Animal Research: Reporting of In Vivo Experiments) (https://arriveguidelines.org).

"Because of the 10- to 100-fold changes in TRH and TRH-like peptide levels in response to the estrus cycle. female rats were not included in the present study" [27].

\section{Effect of acute, chronic and withdrawal treatment with rifaximin in normal rat chow on levels of TRH and TRH-like peptides in rat brain and peripheral tissues} Sixteen young adult male Sprague-Dawley rats, body weight (mean \pm SD) $203 \pm 6$ g, 3.0\% CV, were divided into 4 groups $(\mathrm{n}=4 /$ group$)$. The control $(\mathrm{CON})$ group remained on ad libitum standard Purina powdered rodent chow and water for 10 days until decapitation. The acute (AC) group received ad libitum powdered rodent chow and water for 9 days and then $1 \mathrm{~g}$ rifaximin (Sigma, St. Louis, MO)/500 g powdered rat chow for $24 \mathrm{~h}$. Assuming $16.7 \mathrm{~g}$ chow consumption/day, this would provide $150 \mathrm{mg}$ rifaximin/ $\mathrm{kg}$ body weight for $200 \mathrm{~g}$ rats. The chronic (CHR) animals received RF in powdered chow for 10 days. The withdrawal (WD) rats received RF chow for 8 days and then normal chow for 2 days. The effect of RF withdrawal on TRH and TRH-like peptide levels when compared to the corresponding acute effects can reveal the relative contribution of changes in peptide 
biosynthesis (hours) to changes in peptide release (minutes) [28].

\section{Dissection of rat brain and peripheral tissues}

All rats were decapitated without anesthesia to avoid rapid, anesthetic-induced, blockade of peptide release [29]. Nucleus accumbens (NA), amygdala (AY), frontal cortex (FCX), cerebellum (CBL), medulla oblongata (MED), anterior cingulate (ACNG), posterior cingulate (PCNG), striatum (STR), piriform cortex (PIR), hippocampus $(\mathrm{HC})$, entorhinal cortex (ENT), adrenals $(\mathrm{AD})$, pancreas (PAN), prostate (PR), epididymis (EP), testis $(\mathrm{T})$, heart $(\mathrm{H})$ and liver $(\mathrm{L})$ were hand dissected, weighed rapidly, and then extracted as previously described in detail [30].

\section{Serum hormone assays}

Serum rat leptin, rat insulin, testosterone, free $\mathrm{T}_{4}$, total $\mathrm{T}_{3}$ and glucose were measured (assay range, intra-assay CV\%) with the following commercial RIA kits: rat leptin $(0.801-200 \mathrm{ng} / \mathrm{ml}, 3.2)$ and rat insulin $(0.0329-2.0 \mathrm{ng} / \mathrm{ml}$, 4.8) (Linco Research, Inc., St. Charles, MO), testosterone $(0.05-40 \mathrm{ng} / \mathrm{ml}, 6.7)$, free $\mathrm{T}_{4}(0.045-60 \mathrm{ng} / \mathrm{DL}, 4.6)$ and total $\mathrm{T}_{3}(0.06-80 \mathrm{pg} / \mathrm{ml}, 4.8)$ (MP Biomedical, Solon, $\mathrm{OH})$. Serum glucose was measured with the Contour Next EZ Blood Glucose Monitoring System (Ascensia Diabetes Care US, Inc., Parsippany, NJ).

\section{HPLC and RIA procedures, HPLC peak identification and quantitation}

HPLC and RIA procedures, peak identification, and quantitation by co-chromatography with synthetic TRH and TRH-like peptides, relative potency analysis of multiple antibodies to TRH and TRH-like peptides, and mass spectrometry and have been previously reported in detail [11, 28, 31-33].

Briefly, after boiling, tissues were dried, re-extracted with methanol, dried and defatted by water-ethyl ether partitioning. Dried samples were dissolved in $0.1 \%$ trifluroacetic acid (TFA) and loaded onto reverse phaseC18 Sep-Pak cartridges (Water, Milford, MA). TRH and TRH-like peptides were eluted with 50\% methanol. Dried peptides were again dissolved in TFA, filtered and then fractionated by HPLC using a 4.6-150 mm Econosphere, $3 \mathrm{~mm} \mathrm{C} 18$ reverse phase column (Dr. Maisch $\mathrm{GmbH}$, Ammerbuch, Germany) and a $0.2 \% / \mathrm{min}$ gradient of acetonitrile. The $0.5 \mathrm{ml}$ fractions collected were dried completely and reconstituted with $0.10 \mathrm{ml}$ of $0.02 \% \mathrm{NaN} 3$ just before RIA (Fig. 1).

The antiserum used (8B9) cross-reacts with TRH and nine TRH-like peptides with a relative potency of displacement ranging from 2.31 (Lys-TRH) to 0.288 (SerTRH) relative to Tyr-TRH (Table 2), (see [28]). Two of the regularly observed peaks $(2 \mathrm{a}, \mathrm{b})$ consist of a mixture of unidentified TRH-like peptides. Of the eight observed peptides three have so far been confirmed by mass spectrometry: TRH, Glu-TRH and Tyr-TRH [31]. Tissue samples from the 4 rats within each treatment group were pooled prior to HPLC to provide the minimum amount of immunoreactivity needed for reliable RIA measurements.

The mean recovery of TRH and TRH-like peptide immunoreactivity from all tissues studied was $84 \pm 15 \%$ (mean $\pm \mathrm{SD}$ ). The within-assay and between-assay coefficient of variation for measuring $333 \mathrm{pg} / \mathrm{ml}$ TRH was $4.8 \%$ and $16.9 \%$, respectively. All HPLC fractions obtained from a given brain region or peripheral tissue were analyzed in the same RIA. The minimum detectable dose for TRH was $5 \mathrm{pg} / \mathrm{ml}$. The specific binding of $\left[{ }^{125} \mathrm{I}\right] \mathrm{TRH}$ (Bo/T) was $25 \%$.

\section{Statistical analysis}

"Statistical methods for comparing peak areas were made with the aid of Statview (Abacus Concepts, Inc., Berkeley, CA), a statistical software package for the Macintosh computer. All multi-group comparisons were carried out by one way analysis of variance using post hoc Scheffe contrast with the control group" [30].

"The mean within-group coefficient of variation $(\mathrm{CV})$ (SD/mean, CV-within group) for each tissue and TRH/ TRH-like peptide combination, across four photoperiod intervals, has been previously reported (circadian rhythm experiment) for untreated Sprague-Dawley male rats" [30]. Mean within-group CVs in brain ranged from $4.5 \%$ for TRH levels in AY to 43\% for Phe-TRH in HY, and from $12 \%$ for Val-TRH in testis to $41 \%$ for Trp-TRH in EP for peripheral tissues. These CVs were then used to estimate the level of significance, by on way ANOVA, of changes in the pooled mean values (see [34]) of TRH and TRHlike peptide levels following acute (AC), chronic (CHR) and withdrawal (WD) ingestion of RF" [30]. Pooling of at least 4 tissue extracts was required to provide sufficient signal-to-noise in the RIA for many brain regions and to keep the total number of HPLC fractions to be analyzed reasonable: 4 treatment groups $\times 19$ tissues $\times 100$ HPLC fractions/tissue pool $=7600$ RIA samples for the present study. Without pooling the total number of HPLC fractions would have been $4 \times 7600=30,400$.

\section{Results \\ Body weights}

Mean body weights for all animals at the time of decapitation (9 weeks) was $269 \pm 12$ g, 4.5\% CV. Mean animal weights for each RF treatment group did not differ significantly with the untreated controls by one way ANOVA. 


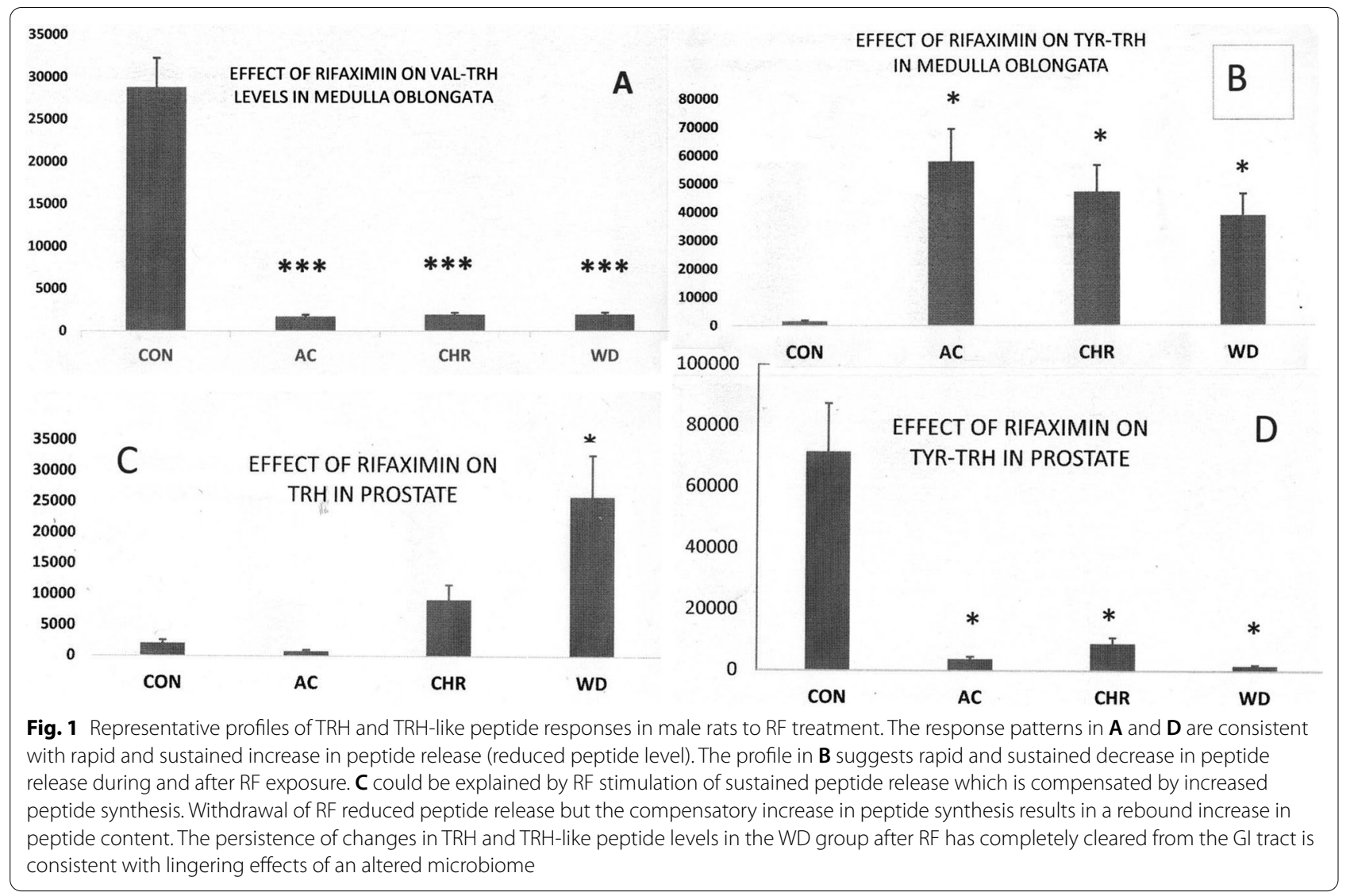

\section{Serum hormone levels following oral rifaximin}

Serum glucose levels for the CHR group were significantly lowers than the WD group $(\mathrm{p}<0.05)$. All other serum hormone levels did not differ significantly between experimental groups by one way ANOVA (Table 1 ).

\section{Overview of TRH and TRH-like peptide data selection and presentation}

Our combined HPLC-RIA methodology can resolve 10 TRH and TRH-like peptides: Glu-TRH, Peaks 2a, b (partially resolved mixture of TRH-like peptides), TRH, Val-TRH, Thr-TRH, Tyr-TRH, Leu-TRH, PheTRH and Trp-TRH [35]. The present study evaluated 12 brain regions and 7 peripheral tissues. This represents $10 \times 19=190$ peptide mean values.

\section{HPLC results in brain and peripheral tissues}

Significant, 25- to 37-fold, increases in Tyr-TRH and 93-94\% decreases in Val-TRH levels in medulla oblongata (all treatment groups, Table 2) and increases in TRH (13-fold, WD group) and Val-TRH (fivefold, WD group) and 99\% decreases in Tyr-TRH concentrations in ventral prostate and a 36-fold increase in liver Tyr-TRH (WD group) (Table 3) were observed following rifaximin administration.

Table 1 Effect of oral rifaximin on serum hormone levels of male rats

\begin{tabular}{lllllll}
\hline & Testosterone $\mathbf{n m o l} / \mathbf{L}$ & $\mathbf{f T}_{\mathbf{3}} \mathbf{~} \mathbf{g g} \mathbf{m l}$ & $\mathbf{f T}_{\mathbf{4}} \mathbf{n g} / \mathbf{d l}$ & Leptin $\mathbf{n g} / \mathbf{m l}$ & Rat insulin $\mathbf{n g} / \mathbf{m l}$ & $\mathbf{G l u c o s e} \mathbf{~ m g / d l}$ \\
\hline CON & $16.8 \pm 9.1$ & $2.61 \pm 0.30$ & $2.94 \pm 0.24$ & $3.97 \pm 1.27$ & $0.15 \pm 0.04$ & $130 \pm 8$ \\
AC & $12.3 \pm 4.8$ & $1.90 \pm 0.35$ & $2.69 \pm 0.49$ & $3.69 \pm 1.88$ & $0.15 \pm 0.09$ & $128 \pm 19$ \\
CHR & $15.5 \pm 4.2$ & $2.43 \pm 0.45$ & $2.72 \pm 0.19$ & $4.43 \pm 1.06$ & $0.21 \pm 0.04$ & $123 \pm 4^{*}$ \\
WD & $14.8 \pm 8.0$ & $2.54 \pm 0.46$ & $3.14 \pm 0.37$ & $2.95 \pm 0.51$ & $0.30 \pm 0.18$ & $145 \pm 16$ \\
\hline
\end{tabular}

There were no significant changes by one way ANOVA versus the corresponding control group. All results are mean \pm SD

${ }^{*} p<0.05$ by one-way ANOVA versus the WD group 
Table 2 Effect of acute (AC), chronic (CHR) and withdrawal (WD) treatments with oral rifaximin on TRH and TRH-like peptide levels in brain regions of male rats $(\mathrm{pg})$

\begin{tabular}{|c|c|c|c|c|c|c|c|c|}
\hline Frontal cortex & Glu-TRH & Peak 2 & TRH & Val-TRH & Tyr-TRH & Leu-TRH & Phe-TRH & Trp-TRH \\
\hline CON & $80 \pm 16$ & $815 \pm 156$ & $1954 \pm 207$ & $1496 \pm 307$ & $3556 \pm 580$ & $1857 \pm 696$ & $1924 \pm 327$ & $736 \pm 161$ \\
\hline$A C$ & $494 \pm 101^{* *}$ & $945 \pm 180$ & $1598 \pm 169$ & $876 \pm 180$ & $1577 \pm 257^{*}$ & $868 \pm 326$ & $1132 \pm 192$ & $521 \pm 114$ \\
\hline $\mathrm{CHR}$ & $188 \pm 39$ & $1143 \pm 218$ & $1867 \pm 198$ & $1485 \pm 304$ & $4283 \pm 698$ & $1761 \pm 660$ & $1487 \pm 253$ & $837 \pm 183$ \\
\hline WD & $395 \pm 81^{*}$ & $874 \pm 167$ & $1310 \pm 139$ & $285 \pm 59^{*}$ & $1515 \pm 247^{*}$ & $904 \pm 339$ & $1087 \pm 185$ & $369 \pm 81$ \\
\hline \multicolumn{9}{|l|}{ Hypothalamus } \\
\hline CON & $657 \pm 217$ & $1771 \pm 638$ & $38,509 \pm 12,323$ & 0 & 0 & $1706 \pm 699$ & $999 \pm 430$ & $616 \pm 172$ \\
\hline$A C$ & $638 \pm 211$ & $1690 \pm 608$ & $34,863 \pm 11,156$ & 0 & 0 & $1368 \pm 561$ & $1138 \pm 489$ & $436 \pm 122$ \\
\hline $\mathrm{CHR}$ & $644 \pm 213$ & $1914 \pm 689$ & $41,487 \pm 13,276$ & 0 & 0 & $1811 \pm 743$ & $943 \pm 405$ & $371 \pm 104$ \\
\hline WD & $841 \pm 278$ & $3983 \pm 1434$ & $28,634 \pm 9163$ & 0 & 0 & $1549 \pm 635$ & $1104 \pm 475$ & $915 \pm 256$ \\
\hline \multicolumn{9}{|l|}{ Amygdala } \\
\hline $\mathrm{CON}$ & $597 \pm 169$ & $1347 \pm 180$ & $513 \pm 23$ & $1312 \pm 125$ & $1543 \pm 262$ & $8408 \pm 2262$ & $1591 \pm 248$ & $1344 \pm 285$ \\
\hline$A C$ & $324 \pm 92$ & $575 \pm 77$ & $1055 \pm 47$ & $980 \pm 93$ & $830 \pm 141$ & $7736 \pm 2081$ & $896 \pm 140$ & $378 \pm 80^{*}$ \\
\hline $\mathrm{CHR}$ & $782 \pm 221$ & $467 \pm 63$ & $1580 \pm 71^{*}$ & $1234 \pm 117$ & $685 \pm 116$ & $9188 \pm 2472$ & $1961 \pm 306$ & $1131 \pm 240$ \\
\hline WD & $410 \pm 116$ & $284 \pm 38^{*}$ & $1012 \pm 46$ & $757 \pm 72$ & $1245 \pm 212$ & $5476 \pm 1473$ & $632 \pm 99$ & $973 \pm 206$ \\
\hline \multicolumn{9}{|l|}{ Hippocampus } \\
\hline $\mathrm{CON}$ & $183 \pm 34$ & $1959 \pm 402$ & $2561 \pm 597$ & $1248 \pm 124$ & $3691 \pm 993$ & $957 \pm 257$ & $2457 \pm 383$ & $1251 \pm 133$ \\
\hline$A C$ & $562 \pm 103^{*}$ & $1097 \pm 225$ & $1349 \pm 314$ & $808 \pm 80$ & $4410 \pm 1186$ & $1536 \pm 413$ & $1188 \pm 185$ & $690 \pm 73$ \\
\hline $\mathrm{CHR}$ & $266 \pm 49$ & $1545 \pm 317$ & $2023 \pm 471$ & $1821 \pm 180$ & $4980 \pm 1340$ & $1930 \pm 519$ & $1343 \pm 210$ & $1167 \pm 124$ \\
\hline WD & $103 \pm 19$ & $1033 \pm 212$ & $1842 \pm 429$ & $1761 \pm 174$ & $8267 \pm 2224$ & $2000 \pm 538$ & $1516 \pm 236$ & $1379 \pm 146$ \\
\hline \multicolumn{9}{|l|}{ Piriform cortex } \\
\hline $\mathrm{CON}$ & $609 \pm 121$ & $860 \pm 127$ & $1324 \pm 207$ & $1100 \pm 155$ & $4284 \pm 878$ & $1576 \pm 345$ & $1856 \pm 380$ & $1203 \pm 383$ \\
\hline$A C$ & $360 \pm 71$ & $1464 \pm 217$ & 0 & $2872 \pm 405^{*}$ & $1055 \pm 216^{*}$ & $822 \pm 180$ & $592 \pm 121^{*}$ & $256 \pm 81^{*}$ \\
\hline $\mathrm{CHR}$ & $131 \pm 26^{*}$ & $515 \pm 76$ & $1104 \pm 172$ & $584 \pm 82$ & $2821 \pm 578$ & $824 \pm 180$ & $865 \pm 177$ & $359 \pm 114^{*}$ \\
\hline WD & $235 \pm 47$ & $176 \pm 26$ & $1268 \pm 198$ & $888 \pm 125$ & $2921 \pm 599$ & $967 \pm 212$ & $610 \pm 125^{*}$ & $203 \pm 65^{*}$ \\
\hline \multicolumn{9}{|c|}{ Nucleus accumbens } \\
\hline CON & $787 \pm 83$ & $3731 \pm 302$ & $17,232 \pm 2188$ & 0 & 0 & $1385 \pm 392$ & $1437 \pm 162$ & $583 \pm 111$ \\
\hline$A C$ & $506 \pm 54$ & $1673 \pm 136$ & $12,653 \pm 1607$ & 0 & 0 & $948 \pm 268$ & $768 \pm 87$ & $300 \pm 57$ \\
\hline $\mathrm{CHR}$ & $394 \pm 42$ & $1304 \pm 106^{*}$ & $5570 \pm 707^{*}$ & 0 & 0 & $861 \pm 244$ & $627 \pm 71$ & $445 \pm 85$ \\
\hline WD & $88 \pm 9^{* *}$ & $486 \pm 39^{*}$ & $3792 \pm 482^{*}$ & 0 & 0 & $162 \pm 46$ & $207 \pm 23^{*}$ & $95 \pm 18^{*}$ \\
\hline \multicolumn{9}{|c|}{ Entorhinal cortex } \\
\hline CON & $63 \pm 13$ & $436 \pm 38$ & $903 \pm 121$ & $652 \pm 83$ & $3511 \pm 572$ & $1497 \pm 222$ & $1461 \pm 346$ & $581 \pm 86$ \\
\hline$A C$ & $214 \pm 45$ & $657 \pm 58$ & $749 \pm 100$ & $803 \pm 102$ & $1770 \pm 289$ & $816 \pm 121$ & $656 \pm 155$ & $397 \pm 59$ \\
\hline $\mathrm{CHR}$ & $244 \pm 52$ & $1275 \pm 112^{*}$ & $2017 \pm 270$ & $1052 \pm 134$ & $3271 \pm 533$ & $1913 \pm 283$ & $1052 \pm 249$ & $1005 \pm 149$ \\
\hline WD & $312 \pm 66^{*}$ & $1269 \pm 112^{*}$ & $1613 \pm 216$ & $1661 \pm 211$ & $6523 \pm 1063$ & $1763 \pm 261$ & $1424 \pm 337$ & $940 \pm 139$ \\
\hline \multicolumn{9}{|l|}{ Striatum } \\
\hline CON & $1724 \pm 207$ & $1992 \pm 294$ & $667 \pm 66$ & $1448 \pm 226$ & $983 \pm 153$ & $9623 \pm 1155$ & $2316 \pm 113$ & $2363 \pm 250$ \\
\hline$A C$ & $534 \pm 64$ & $1565 \pm 232$ & $1522 \pm 151$ & $1280 \pm 200$ & $2642 \pm 412$ & $9292 \pm 1115$ & $1071 \pm 52$ & $1373 \pm 145$ \\
\hline $\mathrm{CHR}$ & $2718 \pm 326$ & $401 \pm 59^{*}$ & $1781 \pm 176$ & $764 \pm 119$ & $2205 \pm 344$ & $14,840 \pm 1781$ & $2720 \pm 133$ & $1459 \pm 155$ \\
\hline WD & $2091 \pm 251$ & $717 \pm 106^{*}$ & $2916 \pm 289^{*}$ & $1983 \pm 309$ & $1227 \pm 191$ & $14,663 \pm 1760$ & $2086 \pm 102$ & $2154 \pm 228$ \\
\hline \multicolumn{9}{|c|}{ Medulla oblongata } \\
\hline CON & $2187 \pm 372$ & $492 \pm 45$ & $5377 \pm 645$ & $28,807 \pm 3860$ & $1555 \pm 308$ & $1908 \pm 500$ & $12,443 \pm 2551$ & $2087 \pm 399$ \\
\hline$A C$ & $3269 \pm 556$ & $347 \pm 32$ & $2263 \pm 272^{*}$ & $1709 \pm 229^{* * *}$ & $58,005 \pm 11,485^{*}$ & $2335 \pm 612$ & $2087 \pm 428^{* *}$ & $915 \pm 175^{*}$ \\
\hline $\mathrm{CHR}$ & $4269 \pm 726$ & $1562 \pm 144^{*}$ & $1293 \pm 155^{* *}$ & $1938 \pm 260^{* * *}$ & $47,265 \pm 9359^{*}$ & $2390 \pm 626$ & $2133 \pm 437^{* *}$ & $715 \pm 137^{*}$ \\
\hline WD & $1916 \pm 326$ & $172 \pm 16$ & $1550 \pm 186^{* *}$ & $1967 \pm 264^{* * *}$ & $38,788 \pm 7680^{*}$ & $1147 \pm 301$ & $1312 \pm 269^{* *}$ & $355 \pm 68^{* *}$ \\
\hline \multicolumn{9}{|l|}{ Cerebellum } \\
\hline $\mathrm{CON}$ & $1636 \pm 128$ & $332 \pm 24$ & $3747 \pm 502$ & $2328 \pm 247$ & $11,040 \pm 1557$ & $3041 \pm 988$ & $2845 \pm 321$ & $2064 \pm 584$ \\
\hline$A C$ & $1539 \pm 120$ & $559 \pm 40$ & $2461 \pm 330$ & $2118 \pm 225$ & $10,117 \pm 1426$ & $2070 \pm 673$ & $1881 \pm 213$ & $1004 \pm 284$ \\
\hline $\mathrm{CHR}$ & - & - & - & - & - & - & - & - \\
\hline WD & $2200 \pm 172$ & $1020 \pm 72$ & $4710 \pm 631$ & $2070 \pm 219$ & $10,320 \pm 1455$ & $2220 \pm 722$ & $5660 \pm 640$ & $1480 \pm 419$ \\
\hline
\end{tabular}


Table 2 (continued)

\begin{tabular}{|c|c|c|c|c|c|c|c|c|}
\hline Anterior & Cingulate & & & & & & & \\
\hline CON & $313 \pm 31$ & $2352 \pm 315$ & $684 \pm 150$ & $579 \pm 115$ & $1769 \pm 276$ & $993 \pm 337$ & $997 \pm 254$ & $385 \pm 76$ \\
\hline$A C$ & $178 \pm 18$ & $545 \pm 73^{*}$ & $895 \pm 196$ & $1055 \pm 209$ & $1945 \pm 303$ & $666 \pm 226$ & $544 \pm 139$ & $240 \pm 48$ \\
\hline $\mathrm{CHR}$ & $202 \pm 20$ & $776 \pm 104$ & $1076 \pm 236$ & $750 \pm 149$ & $2009 \pm 313$ & $722 \pm 245$ & $934 \pm 238$ & $586 \pm 116$ \\
\hline WD & $207 \pm 20$ & $398 \pm 53^{* *}$ & $700 \pm 153$ & $536 \pm 106$ & $1387 \pm 216$ & $200 \pm 68$ & $663 \pm 169$ & $223 \pm 44$ \\
\hline Posterior & Cingulate & & & & & & & \\
\hline CON & $27 \pm 6$ & $521 \pm 100$ & $1967 \pm 209$ & $912 \pm 187$ & $4697 \pm 766$ & $1676 \pm 629$ & $1217 \pm 207$ & $353 \pm 77$ \\
\hline$A C$ & $138 \pm 28^{*}$ & $944 \pm 180$ & $1319 \pm 140$ & $885 \pm 181$ & $3286 \pm 536$ & $976 \pm 366$ & $950 \pm 162$ & $943 \pm 207$ \\
\hline $\mathrm{CHR}$ & $197 \pm 40^{*}$ & $1486 \pm 284$ & $1659 \pm 176$ & $1093 \pm 224$ & $4470 \pm 729$ & $1465 \pm 549$ & $1151 \pm 196$ & $753 \pm 165$ \\
\hline WD & $116 \pm 24$ & $1403 \pm 268$ & $1616 \pm 172$ & $1818 \pm 373$ & $13,661 \pm 2227$ & $1776 \pm 666$ & $1870 \pm 318$ & $676 \pm 148$ \\
\hline
\end{tabular}

All results are mean $\pm S D$

${ }^{*} \mathrm{p}<0.05 ;{ }^{* *} \mathrm{p}<0.01 ;{ }^{* * *} \mathrm{p}<0.002$ by one way ANOVA using post hoc Scheffe contrasts versus the control group

Table 3 Effect of acute (AC), chronic (CHR) and withdrawal (WD) treatments with rifaximin on TRH and TRH-like peptide levels in peripheral tissues (pg)

\begin{tabular}{|c|c|c|c|c|c|c|c|c|}
\hline Prostate & Glu-TRH & Peak 2 & TRH & Val-TRH & Tyr-TRH & Leu-TRH & Phe-TRH & Trp-TRH \\
\hline CON & $1898 \pm 645$ & $48,829 \pm 15,625$ & $2068 \pm 765$ & $12,830 \pm 3464$ & $71,618 \pm 22,202$ & $9626 \pm 3562$ & $9435 \pm 2925$ & $4285 \pm 1328$ \\
\hline$A C$ & $1993 \pm 678$ & $39,195 \pm 12,542$ & $800 \pm 296$ & $8922 \pm 2409$ & $3748 \pm 1162^{*}$ & $13,415 \pm 4964$ & $47,016 \pm 14,575^{*}$ & $2981 \pm 924$ \\
\hline $\mathrm{CHR}$ & $1827 \pm 621$ & $33,166 \pm 10,613$ & $9192 \pm 3401$ & $41,357 \pm 11,166$ & $8858 \pm 2746^{*}$ & $4079 \pm 1509$ & $8103 \pm 2512$ & $3971 \pm 1231$ \\
\hline WD & $3215 \pm 1093$ & $54,959 \pm 17,587$ & $25,935 \pm 9596^{*}$ & $64,372 \pm 17,380^{*}$ & $1640 \pm 508^{*}$ & $4200 \pm 1554$ & $10,188 \pm 3158$ & $4346 \pm 1347$ \\
\hline \multicolumn{9}{|l|}{ Liver } \\
\hline CON & $277 \pm 66$ & $216 \pm 41$ & $584 \pm 228$ & $361 \pm 105$ & $58 \pm 17$ & $765 \pm 245$ & $452 \pm 145$ & $327 \pm 75$ \\
\hline$A C$ & $426 \pm 102$ & $313 \pm 59$ & $954 \pm 372$ & $394 \pm 114$ & $36 \pm 11$ & $1390 \pm 445$ & $930 \pm 298$ & $708 \pm 163$ \\
\hline $\mathrm{CHR}$ & $586 \pm 141$ & $385 \pm 73$ & $1919 \pm 748$ & $2050 \pm 595^{*}$ & $230 \pm 69$ & $537 \pm 172$ & $923 \pm 295$ & $360 \pm 83$ \\
\hline WD & $626 \pm 150$ & $224 \pm 43$ & $1427 \pm 557$ & $738 \pm 214$ & $2073 \pm 622^{*}$ & $571 \pm 183$ & $1167 \pm 373$ & $451 \pm 104$ \\
\hline \multicolumn{9}{|l|}{ Testis } \\
\hline CON & $43 \pm 10$ & $260 \pm 99$ & $714 \pm 150$ & $448 \pm 54$ & $2228 \pm 646$ & $656 \pm 190$ & $610 \pm 140$ & $174 \pm 52$ \\
\hline$A C$ & $93 \pm 21$ & $68 \pm 26$ & $370 \pm 78$ & $515 \pm 62$ & $2343 \pm 679$ & $807 \pm 234$ & $623 \pm 143$ & $448 \pm 134$ \\
\hline $\mathrm{CHR}$ & $275 \pm 63^{*}$ & $218 \pm 83$ & $693 \pm 146$ & $716 \pm 86$ & $3005 \pm 871$ & $957 \pm 278$ & $1190 \pm 274$ & $521 \pm 156$ \\
\hline WD & $83 \pm 19$ & $160 \pm 61$ & $392 \pm 82$ & $460 \pm 55$ & $961 \pm 279$ & $516 \pm 150$ & $577 \pm 133$ & $442 \pm 133$ \\
\hline \multicolumn{9}{|l|}{ Heart } \\
\hline CON & $181 \pm 54$ & $134 \pm 24$ & $427 \pm 51$ & $408 \pm 126$ & $104 \pm 31$ & $533 \pm 139$ & $575 \pm 196$ & $271 \pm 98$ \\
\hline$A C$ & $170 \pm 51$ & $86 \pm 15$ & $242 \pm 29$ & $114 \pm 35$ & $159 \pm 48$ & $233 \pm 61$ & $247 \pm 84$ & $195 \pm 70$ \\
\hline $\mathrm{CHR}$ & $123 \pm 37$ & $102 \pm 18$ & $271 \pm 33$ & $344 \pm 107$ & $73 \pm 22$ & $304 \pm 79$ & $776 \pm 264$ & $415 \pm 149$ \\
\hline WD & $381 \pm 114$ & $203 \pm 37$ & $312 \pm 37$ & $137 \pm 42$ & $283 \pm 85$ & $227 \pm 59$ & $422 \pm 143$ & $307 \pm 111$ \\
\hline \multicolumn{9}{|l|}{ Pancreas } \\
\hline CON & $234 \pm 82$ & $279 \pm 95$ & $139 \pm 58$ & $206 \pm 47$ & $214 \pm 45$ & $870 \pm 200$ & $464 \pm 97$ & $531 \pm 117$ \\
\hline$A C$ & $191 \pm 67$ & $243 \pm 83$ & $115 \pm 48$ & $175 \pm 40$ & $165 \pm 35$ & $503 \pm 116$ & $419 \pm 88$ & $319 \pm 70$ \\
\hline $\mathrm{CHR}$ & $101 \pm 35$ & $313 \pm 106$ & $173 \pm 73$ & $168 \pm 39$ & $114 \pm 24$ & $508 \pm 117$ & $1085 \pm 228^{*}$ & $620 \pm 136$ \\
\hline WD & $344 \pm 120$ & $238 \pm 81$ & $64 \pm 27$ & $75 \pm 17$ & $80 \pm 17^{*}$ & $241 \pm 55^{*}$ & $398 \pm 84$ & $523 \pm 115$ \\
\hline \multicolumn{9}{|l|}{ Adrenals } \\
\hline CON & $588 \pm 188$ & $1905 \pm 286$ & $1577 \pm 315$ & $1169 \pm 339$ & $2109 \pm 970$ & $1432 \pm 473$ & $1877 \pm 507$ & $1101 \pm 374$ \\
\hline$A C$ & $484 \pm 155$ & $1194 \pm 179$ & $856 \pm 171$ & $934 \pm 271$ & $1641 \pm 755$ & $535 \pm 177$ & $1288 \pm 348$ & $1145 \pm 389$ \\
\hline $\mathrm{CHR}$ & $403 \pm 129$ & $835 \pm 125^{*}$ & $871 \pm 174$ & $564 \pm 164$ & $1646 \pm 757$ & $616 \pm 203$ & $701 \pm 189$ & $216 \pm 73$ \\
\hline WD & $74 \pm 24$ & $124 \pm 19^{* *}$ & $692 \pm 138^{*}$ & $523 \pm 152$ & $631 \pm 290$ & $198 \pm 65^{*}$ & $448 \pm 121$ & $124 \pm 42$ \\
\hline
\end{tabular}

All results are mean $\pm S D$

${ }^{*} p<0.05$; ${ }^{* *} p<0.01$ by one way ANOVA using post hoc Scheffe contrasts versus the control group 
The number of significant changes in TRH and TRHlike peptide levels in brain resulting from RF treatment (In parentheses), in descending order were: MED (16), PIR (8), NA (7), FCX (5), STR (3), AY (3), ENT (3), ACNG (2), PCNG (2), HC (1), HY (0) and CBL (0) as seen in Table 2 . The corresponding ranking for peripheral tissues were: PR (6), AD (4), PAN (3), L (2), T (1), H (0), (see Table 3). The pooled EP controls were lost during extraction so results for this tissue could not be analyzed.

\section{Discussion}

Acute, chronic and withdrawal treatment with RF resulted in significant decreases in TRH, Val-TRH, PheTRH and Trp-TRH and marked increases in Tyr-TRH levels in the MED (Table 2). These changes result from alterations in the biosynthesis and release of these tripeptides. The rapidity of these responses is consistent with increased TRH, Val-TRH, Phe-TRH and Trp-TRH and decreased Tyr-TRH release, respectively [32]. These remarkable changes in peptide levels within the MED is consistent with current knowledge regarding the role of TRH (and TRH-like peptides) as mediators of brain-gut communication via the vagus nerve $[10,36]$. The antidepressant activity of Tyr-TRH [31] and analeptic effect of Val-TRH [37] correspond with actions of TRH [11]. TRH and TRH-like peptide biosynthesis occurs within large dense core vesicles (LDCV) of glutamatergic neurons [32, 33]. They are co-released with glutamate and act to moderate the effects of this excitotoxic neurotransmitter [7, 8]. Neuropeptides, such as TRH, which are co-released with classical neurotransmitters are now considered primary mediators of brain circuit connectivity with a longer duration of action [38].

Dysbiosis of the microbiome has been implicated in prostatitis and prostate cancer [12, 39]. Rifaximin, an antibiotic which does not cross the blood-gut barrier, is currently being evaluated as a treatment for these pathologies [40]. It is noteworthy that among the peripheral tissues analyzed, PR had the highest number of significant changes in TRH and TRH-like peptide levels (Table 3) in response to RF treatment. PR has very high levels of TRH and TRH-like peptides which are subject to marked circadian rhythmicity [11]. TRH stimulates the adenylyl cyclase in basal cell membranes of the rat ventral prostate [41].

Withdrawal of RF increased TRH and Val-TRH levels in prostate (Table 3), which is consistent with RF stimulation of both biosynthesis and release of these peptides. Because the reduction in the peptide release rate is rapid but the changes in RF-stimulated peptide synthesis is slow with RF withdrawal, a rebound in the levels of these peptides is observed. Acute RF treatment increased Phe-TRH levels consistent with a rapid inhibition of release for this peptide in response to RF followed by a compensatory decrease in biosynthesis returning CHR Phe-TRH levels to $\mathrm{CON}$ values. AC, CHR and WD treatment with RF all decreased TyrTRH levels significantly in prostate (Table 3). These observations may reveal a rapid and sustained increase in Tyr-TRH release in response to RF treatment which is not accompanied by marked changes in the processing of Tyr-TRH progenitor peptides.

Withdrawal of RF resulted in significant decreases in the levels of all TRH and TRH-like peptides measured in the adrenals (Table 3). This is consistent with an acute decrease in biosynthesis and/or increase in release of these peptides [32]. RF has modest, transient, and beneficial effects on stress-related changes in the gut microbiome, inflammation, permeability and hyperalgesia as well as central responses to social stress [15, 17, 18, 42]. Manipulation of the gut microbiome can have significant effects on cortisol levels in urine [1]. The influence of the microbiota on the function of the HPA axis which regulates corticosterone levels was first demonstrated by Sudo et al. [43]. Germ-free mice have reduced levels of brainderived neurotrophic factors in the cortex, hippocampus, and amygdala, and altered expression of glutamate and dopamine receptors in specific regions of the brain [44].

Administration of the probiotic bacterium Lactobacillus rhamnosus strain JB- $1^{\mathrm{TM}}$ to mice significantly altered the expression of the gene coding for a GABA receptor in multiple regions of the brain, including the amygdala, hippocampus, and cortex. This effect was abolished by vagotomy [45].

The vagus nerve is the principal neuronal link between internal organs and the brain and has now been shown to be integral to the regulation of an array of autonomic functions, such as breathing, heart functions, pancreatic and liver regulation of metabolism, modulation of immune and inflammatory responses via the spleen, mood, and even consciousness [46, 47].

The TRH-degrading serum enzyme is a product of liver [48]. It rapidly metabolizes TRH and most TRHlike peptides in the circulation except Glu-TRH [49]. Rifaximin is used for the prevention of recurrent overt hepatic encephalopathy [50]. Significant increases in Val-TRH and Tyr-TRH levels were observed in liver in response to RF treatment (Table 3). Functional brain MRI studies of the responses of patients with cirrhosis to rifaximin treatment reveal higher activation in various brain regions including the frontal cortex, hippocampus, anterior and posterior cingulate [51]. RF is also utilized for the treatment of irritable bowel disease, diverticular disease, and small bowel bacterial overgrowth $[16,17,50]$. RF modulates inflammatory cytokines and intestinal permeability [52]. Medullary 
TRH and gastric vagal efferent and afferent circuits play a crucial role in the modulation of gastric integrity [53].

\section{Conclusions}

The marked responsivity of TRH and TRH-like peptide expression to RF-induced alterations in gut microbiota of normal rats is consistent with the participation of these peptides in vagally-mediated brain-gut signaling. The observed effects persist after RF, which does not cross the blood-gut barrier, has cleared the GI tract. We expect future studies will extend this concept to antidepressant, anxiolytic, anti-obesity, GI-, liver- and prostate-protective effects of rifaximin [54].

\section{Acknowledgements}

The authors thank the VAGLAHS Research Service for the provision of laboratory and office space and equipment for the performance of the present studies.

\section{Authors' contributions}

AEP: developed the HPLC and RIA methodology, conceived the current study, performed the daily handling of rats, prepared the powdered diet, with and without added rifaximin, dissected peripheral tissues and hypothalamus, carried out the extraction and analysis of TRH and TRH-like peptide levels by a combination of HPLC and RIA, integrated peptide peak areas, statistically analyzed results, and wrote manuscript. AS performed the rat brain dissections and edited the manuscript. All authors read and approved the final manuscript.

\section{Funding}

This research did not receive any specific Grant from funding agencies in the public, commercial, or not-for-profit sectors.

\section{Availability of data and materials}

All statistically summarized data are included in this published article. Primary data available from AEP upon reasonable request.

\section{Declarations}

Ethics approval and consent to participate

The present studies were approved by the VA Greater Los Angeles Healthcare System (VAGLAHS) Institutional Animal Care and Use Committee (IACUC) under Protocol No. 030090-10 on June 21, 2018. Experiments were conducted according to the Guide for the Care and Use of Laboratory Animals, 2011. The ARRIVE guidelines have been followed in the design, execution, analysis and presentation of the results.

\section{Consent for publication}

Not applicable.

\section{Competing interests}

There are no competing interests, financial or nonfinancial, to declare.

\section{Author details}

${ }^{1}$ Research Services, VA Greater Los Angeles Healthcare System, Bldg. 114, Rm. 229B, 11301 Wilshire Blvd., Los Angeles, CA 90073, USA. Psychiatry Services, VA Greater Los Angeles Healthcare System, Los Angeles CA 90073, USA.

${ }^{3}$ Center for Ulcer Research and Education, VA Greater Los Angeles Healthcare System, Los Angeles CA 90073, USA. ${ }^{4}$ Departments of Psychiatry and Biobehavioral Sciences, University of California, Los Angeles, CA 90073, USA. ${ }^{5}$ Brain Research Institute, University of California, CA 90073 Los Angeles, USA.

${ }^{6}$ Department of Medicine, University of California, Los Angeles, CA 90073, USA.
Received: 5 October 2021 Accepted: 11 February 2022

Published online: 21 February 2022

\section{References}

1. Douglas AE. Chapter 5. Microbial drivers of animal behavior. In: Fundamentals of microbiome science. How microbes shape animal biology. Princeton University Press: Princeton; 2018. p. 93-120.

2. Hill-Yardin EL, Grabrucker AM, Franks AE, Luna RA, Monif M. Editorial: interactions of the nervous system with bacteria. Front Neurosci. 2021. https://doi.org/10.3389/fnins.2021.682744.

3. Lach G, Schellekens H, Dinan TG, Cryan JF. Anxiety, depression, and the microbiome: a role for gut peptides. Neurotherapeutics. 2018;15:36-59.

4. Martin CR, Osadchiy V, Kalani A, Mayer EA. The brain-gut-microbiome axis. Cell Mol Gastroenterol Hepatol. 2018;6:133-48.

5. Sarkar A, Harty S, Johnson KVA, Moeller AH, Carmody RN, Lehto SM, Erdman SE, Dunbar RIM, Burnet WJ. The role of the microbiome in the neurobiology of social behaviour. Biol Rev. 2020. https://doi.org/10.1111/ brv.12603.

6. Jones S. A new villain in neuronal death. A newly discovered partnership for glutamate triggers brain toxicity. Science. 2020;370:168-9.

7. Koenig ML, Sgarlat CM, Yourick DL, Long JB, Meyerhoff JL. In vitro neuroprotection against glutamate-induced toxicity by pGlu-Glu-Pro- $\mathrm{NH}_{2}$ (EEP). Peptides. 2001;22:2091-7.

8. Koenig ML, Yourick DL, Meyerhoff JL. Thyrotropin-releasing hormone $(\mathrm{TRH})$ attenuates glutamate-stimulated increases in calcium in primary neuronal cultures. Brain Res. 1996:730:143-9.

9. Yan J, Bengtson P, Buchthal B, Hageston AM, Bading H. Coupling of NMDA receptors and TRPM4 guides discovery of unconventional neuroprotectants. Science. 2020;370:191

10. Tache $Y$, Adelson D, Yang $H$. TRH/TRH-R1 receptor signaling in the brain medulla as a pathway of vagally mediated gut responses during the cephalic phase. Curr Pharm Des. 2014;20:2725-30.

11. Pekary AE, Sattin A. TRH and TRH-like peptide levels co-vary with reproductive and metabolic rhythms. Horm Metab Res. 2017;49:86-94.

12. Porter CM, Shrestha E, Peiffer LB, Sfanos KS. The microbiome in prostate inflammation and prostate cancer. Prostate Cancer Prostatic Dis. 2018:21:345-54.

13. Li H, Xiang Y, Zhu Z, Wang W, Jiang Z, Zhao M, et al. Rifaximin-mediated gut microbiota regulation modulates the function of microglia and protects against CUMS-induced depression-like behaviors in adolescent rat. J Neuroinflammation. 2021;18:254. https://doi.org/10.1186/ s12974-021-02303-y.

14. Tamaoki S, Suzuki H, Okada M, Fukui N, Isobe M, Saito T. Development of an experimental rat model of hyperammonemic encephalopathy and evaluation of the effects of rifaximin. Eur J Pharmacol. 2016;779:168-76.

15. Wang $H$, Braun $C$, Enck P. Effects of rifaximin on central responses to social stress-a pilot experiment. Neurotherapeutics. 2018;15:807-18.

16. Xu D, Gao J, Gilliland M 3rd, Wu X, Song I, Kao JY, et al. Rifaximin alters intestinal bacteria and prevents stress-induced gut inflammation and visceral hyperalgesia in rats. Gastroenterology. 2014;146:484-96.

17. Jin Y, Ren X, Li G, Li Y, Zhang L, Wang H, Qian W, Hou X. Beneficial effects of rifaximin in post-infectious irritable bowel syndrome mouse model beyond gut microbiota. J Gastroenterol Hepatol. 2018;33:443-52.

18. Fodor AA, Pimentel M, Chey WD, Lembo A, Golden PL, Israel RJ, Carroll IM. Rifaximin is associated with modest, transient decreases in multiple taxa in the gut microbiota of patients with diarrhoea-predominant irritable bowel syndrome. Gut Microbes. 2019;10:22-33.

19. Barnes MJ, Rogers RD, Van Meter MJ, Hermann GE. Co-localization of TRHR1 and LepRb receptors on neurons in the hindbrain of the rat. Brain Res. 2010;1355:70-85.

20. Lang UE, Beglinger C, Schweinfurth N, Walter M, Borgwardt S. Nutritional aspects of depression. Cell Physiol Biochem. 2015;37:1029-43.

21. Michael DR, Jack AA, Masetti G, Davies TS, Loxley KE, Kerry-Smith J, et al. A randomized controlled study shows supplementation of overweight and obese adults with lactobacilli and bifidobacterial reduces bodyweight and improves well-being. Sci Rep. 2020;10:4183. https://doi.org/10.1038/ s41598-020-60991-7. 
22. Racz B, Duskova M, Starka L, Hainer V, Kunesova M. Link between the circadian rhythm, obesity and the microbiome. Physiol Res. 2018;67(Suppl 3):S409-20

23. Tomiyama AJ. Stress and obesity. Annu Rev Psychol. 2019;70:703-18.

24. Pekary AE, Sattin A, Blood JD. Rapid modulation of TRH and TRH-like peptide release in rat brain and peripheral tissues by leptin. Brain Res. 2010;1345:9-18.

25. Pekary AE, Sattin A. Rapid modulation of TRH and TRH-like peptide release in rat brain and peripheral tissues by ghrelin and 3-Trp-ghrelin. Peptides. 2012;36:157-67.

26. MacLusky NJ. Euthanasia in endocrinology: the choices get more complex. Endocrinology. 2009;150:2505-6.

27. Pekary AE, Sattin A. Increased TRH and TRH-like peptide release in rat brain and peripheral tissues during proestrus/estrus. Peptides. 2014;52:1-10.

28. Pekary AE, Sattin A, Meyerhoff JL, Chilingar M. Valproate modulates $\mathrm{TRH}$ receptor, $\mathrm{TRH}$ and $\mathrm{TRH}$-like peptide levels in rat brain. Peptides. 2004:25:647-58.

29. Mannisto PT. Central regulation of thyrotropin secretion in rats: methodological aspects, problems and some progress. Med Biol. 1983;61:92-100.

30. Pekary AE, Stevens SA, Sattin A. Circadian rhythms of TRH-like peptide levels in rat brain. Brain Res. 2006;1125:67-76.

31. Pekary AE, Faull KF, Paulson M, Lloyd RL, Sattin A. TRH-like antidepressant peptide, pyroglutamyltyrosylprolineaminde, occurs in rat brain. J Mass Spectrom. 2005;40:1232-6.

32. Pekary AE, Steven SA, Blood JD, Sattin A. Rapid modulation of TRH and TRH-like peptide release in rat brain, pancreas, and testis by a GSK-3 $\beta$ inhibitor. Peptides. 2010;31:1083-93.

33. Pekary AE, Stevens SA, Sattin A. Valproate and copper accelerate TRH-like peptide synthesis in male rat pancreas and reproductive tissues. Peptides. 2006;27:2901-11.

34. Bowker AH, Lieberman GJ. Engineering statistics. Chapter VII. Tests of the hypothesis that the means of two normal distributions are equal when both standard deviations are known. Englewood Cliffs: Prentice-Hall Inc.; 1959. p. 156-210.

35. Pekary AE, Sattin A, Lloyd RL. Ketamine modulates TRH and TRH-like peptide turnover in brain and peripheral tissues of male rats. Peptides. 2015;69:66-7.

36. Ishikawa T, Yang H, Tache Y. Medullary sites of action of the TRH analogue, RX 77368, for stimulation of gastric acid secretion in the rat. Gastroenterology. 1988;95:1470-6.

37. Hinkle PM, Pekary AE, Senanayaki S, Sattin A. Role of TRH receptors as possible mediators of analeptic actions of TRH-like peptides. Brain Res. 2002;935:59-64.

38. Guillaumin MCC, Burdakov D. Neuropeptides as primary mediators of brain circuit connectivity. Front Neurosci. 2021. https://doi.org/10.3389/ fnins.2021.644313.

39. McCulloch JA, Trinchieri G. Gut bacteria enable prostate cancer growth. Testosterone-synthetizing gut bacteria drive resistance to therapy. Science. 2021;374:154-5.

40. Vicari E, Salemi M, Sidoti G, Malaguarnera M, Castiglione R. Symptom severity following rifaximin and the probiotic VSL\#3 in patients with chronic pelvic pain syndrome (due to inflammatory prostatitis) plus irritable bowel syndrome. Nutrients. 2017;9:1208. https://doi.org/10.3390/ nu9111208.

41. Purvis K, Brekke I, Attramadal A, Gordeladze JO. Thyrotropin-releasing hormone (TRH) activates the adenylyl cyclase of nonsecretory cells in the rat ventral prostate. Prostate. 1984;5:613-9.

42. Kuti D, Winkler Z, Horvath K, Juhasz B, Paholcsek M, Stagel A, et al. Gastrointestinal (non-systemic) antibiotic rifaximin differentially affects chronic stress-induced changes in colon microbiome and gut permeability without effect on behavior. Brain Behav Immun. 2020;84:218-28.

43. Sudo N, Chida Y, Aiba Y, Sonoda J, Oyama N, Yu XN, et al. Postnatal microbial colonization programs the hypothalamic-pituitary-adrenal system for stress response in mice. J Physiol. 2004;558:263-75.

44. Luczynski P, McVey Neufeld KA, Oriach CS, Clarke G, Dinan TG, Cryan JF. Growing up in a bubble: using germ-free animals to assess the influence of the gut microbiota on brain and behavior. Int J Neuropsychopharmacol. 2016;19:pyw020.

45. Bravo JA, Forsythe P, Chew MV, Escaravage E, Savignac HM, Dinan TG, et al. Ingestion of Lactobacillus strain regulates emotional behavior and central GABA receptor expression in a mouse via the vagus nerve. Proc Natl Acad Sci USA. 2011;108:16050-5.

46. Fitchett A, Mastitskaya S, Aristovich K. Selective neuromodulation of the vagus nerve. Front Neurosci. 2021. https://doi.org/10.3389/fnins.2021. 685872.

47. Underwood E. A sense of self: communication between the brain and other organs shapes how we think, remember, and feel. Science. 2021;372:1142-5

48. Schmitmeier S, Thole H, Bader A, Bauer K. Purification and characterization of the thyrotropin-releasing hormone (TRH)-degrading serum enzyme and its identification as a product of liver origin. Eur J Biochem. 2002;269:1278-86.

49. Charli J-L, Rodriguez-Rodriguez A, Ortega $\mathrm{KH}$, Cote-Velez A, Uribe RM, Jaimes-Hoy $L$, et al. The thyrotropin-releasing hormone-degrading ectoenzyme, a therapeutic target? Front Pharmacol. 2020;11:640. https://doi. org/10.3389/fphar.2020.00640.eCollection.

50. Shayto RH, Mrad RA, Sharara Al. Use of rifaximin in gastrointestinal and liver diseases. World J Gastroenterol. 2016;22:6638-51.

51. Ahluwalia V, Wade JB, Heuman DM, Hammeke TA, Sanyal AJ, Sterling RK, et al. Enhancement of functional connectivity, working memory and inhibitory control on multi-modal brain MR imaging with rifaximin in cirrhosis: implications for the gut-liver-brain axis. Metab Brain Dis. 2014;29:1017-25.

52. Chey WD, Shah ED, Dupont HL. Mechanism of action and therapeutic benefit of rifaximin in patients with irritable bowel syndrome: a narrative review. Therap Adv Gastroenterol. 2020;23(13):1756284819897531.

53. Kaneko $H$, Tache $Y$, Kusugami K. Importance of medullary thyrotropinreleasing hormone in brain-gut circuits regulating gastric integrity: preclinical studies. J Gastroenterol Suppl. 2002;14:128-32.

54. Strandwitz P. Neurotransmitter modulation by the gut microbiota. Brain Res. 2018;1693(Pt B):128-33.

\section{Publisher's Note}

Springer Nature remains neutral with regard to jurisdictional claims in published maps and institutional affiliations.

Ready to submit your research? Choose BMC and benefit from

- fast, convenient online submission

- thorough peer review by experienced researchers in your field

- rapid publication on acceptance

- support for research data, including large and complex data types

- gold Open Access which fosters wider collaboration and increased citations

- maximum visibility for your research: over $100 \mathrm{M}$ website views per year

At BMC, research is always in progress.

Learn more biomedcentral.com/submissions 